\title{
Multicentre, double-blind, crossover trial to identify the Optimal Pathway for TreatIng neurOpathic paiN in Diabetes Mellitus (OPTION-DM): study protocol for a randomised controlled trial
}

Dinesh Selvarajah', Jennifer Petrie ${ }^{2^{*}}$ D, David White ${ }^{2}$, Steven Julious ${ }^{3}$, Oscar Bortolami $^{2}$, Cindy Cooper ${ }^{2}$, Mike Bradburn², Amanda Loban², Helen Bowler ${ }^{4}$, Lizzie Swaby ${ }^{2}$, Katie Sutherland ${ }^{2}$, Solomon Tesfaye ${ }^{4}$ and on behalf of the OPTION-DM group

\begin{abstract}
Background: The number of people with diabetes is growing rapidly. Diabetes can cause nerve damage leading to severe pain in the feet, legs and hands, which is known as diabetic peripheral neuropathic pain (DPNP). In the UK, the National Institute for Health and Care Excellence (NICE) recommends amitriptyline, duloxetine, pregabalin or gabapentin as initial treatment for DPNP. If this is not effective, adding one of the other drugs in combination with the first is recommended. NICE points out that these recommendations are not based on robust evidence. The OPTION-DM randomised controlled trial has been designed to address this evidence deficit, with the aims of determining the most clinically beneficial, cost-effective and tolerated treatment pathway for patients with DPNP.

Methods/design: A multicentre, double-blind, centre-stratified, multi-period crossover study with equal allocation to sequences (1:1:1:1:1:1) of treatment pathways. Three hundred and ninety-two participants will be recruited from secondary care DPNP centres in the UK. There are three treatment pathways: amitriptyline supplemented with pregabalin, pregabalin supplemented with amitriptyline and duloxetine supplemented with pregabalin. All participants will receive all three pathways and randomisation will determine the order in which they are received. The primary outcome is the difference between 7-day average 24-h pain scores on an 11-point NRS scale measured during the final follow-up week of the treatment pathway. Secondary outcomes for efficacy, cost-effectiveness, safety, patient-perceived tolerability and subgroup analysis will be measured at week 6 and week 16 of each pathway.

Discussion: The study includes direct comparisons of the mainstay treatment for DPNP. This novel study is designed to examine treatment pathways and capture clinically relevant outcomes which will make the results generalisable to current clinical practice. The study will also provide information on health economic outcomes and will include a subgroup study to provide information on whether patient phenotypes predict response to treatment.
\end{abstract}

Trial registration: ISRCTN17545443. Registered on 12 September 2016.

Keywords: Diabetes, Painful diabetic neuropathy, Pregabalin, Duloxetine, Amitriptyline, Crossover trial

\footnotetext{
* Correspondence: j.petrie@sheffield.ac.uk

${ }^{2}$ Clinical Trials Research Unit, University of Sheffield, Sheffield, UK

Full list of author information is available at the end of the article
}

(c) The Author(s). 2018 Open Access This article is distributed under the terms of the Creative Commons Attribution 4.0 International License (http://creativecommons.org/licenses/by/4.0/), which permits unrestricted use, distribution, and reproduction in any medium, provided you give appropriate credit to the original author(s) and the source, provide a link to the Creative Commons license, and indicate if changes were made. The Creative Commons Public Domain Dedication waiver (http://creativecommons.org/publicdomain/zero/1.0/) applies to the data made available in this article, unless otherwise stated. 


\section{Background}

In August 2015, Diabetes UK announced that the prevalence of diabetes had increased by $60 \%$ over the previous decade to 3.3 million. Diabetic peripheral neuropathic pain (DPNP) is a serious complication affecting up to $20-26 \%$ of these patients $[1,2]$. With the prevalence of diabetes set to increase by epidemic proportions over the next decade, DPNP will pose a major treatment challenge $[3,4]$. With advanced disease the pain can extend above the feet and may involve the whole of the legs, and when this is the case there is often upper limb involvement also. Moderate-to-severe unremitting lower limb pain is present in over $70 \%$ of sufferers $[2,5]$ and causes insomnia, poor Quality of Life (QoL), unemployment and depression [6-9].

The mainstay of treatment for DPNP is pharmacotherapy. Recent National Institute for Health and Care Excellence (NICE) guidance (173) [10] recommends a choice of amitriptyline, duloxetine, pregabalin or gabapentin as initial treatment. All are licensed treatments for DPNP except amitriptyline, which has been used off-license for more than 25 years. There is moderate evidence for the efficacy of each drug based on Cochrane reviews [11-14] and meta-analyses [15-17], but the best we can hope for any monotherapy is $50 \%$ pain relief in $50 \%$ of patients [10]. This is often accompanied by side effects (dry mouth, constipation, sedation, dizziness, falls, nausea, oedema, etc.) in around $10-20 \%$ depending on dose. NICE recommends combination treatment if initial treatment is not effective (the majority) [10]. However, as NICE points out recommendations are not based on robust evidence as: (1) there are few well-designed head-to-head studies comparing the first-line drugs and their combinations; (2) most studies were flawed with inadequate power, inappropriate endpoints, short duration of follow-up and (3) many randomised controlled trials (RCTs) lacked appropriate health-related QoL (HRQL) measures including functionality and failed to measure impact of drug-related adverse effects on health economics and QoL [10]. An RCT is needed to address these deficiencies.

The aims of the OPTION-DM study will be to determine the most clinically beneficial, cost-effective and tolerated treatment pathway for patients with DPNP. The study has been designed to have direct clinical applicability in the management of DPNP following completion.

\section{Study objectives}

1. To evaluate if at least one of the three pathways is superior to the other pathways in terms of pain symptoms, quality of life and cost-effectiveness

2. To evaluate if at least one monotherapy is superior to a different monotherapy in improving pain symptoms
3. To describe Adverse Event and Serious Adverse Event data for the different treatment pathways for DPNP

4. To conduct an exploratory analysis to investigate whether there are patient phenotypes that predict response to treatment

\section{Methods/design \\ Study design}

OPTION-DM is a multicentre, double-blind, centrestratified, multi-period crossover study with equal allocation to sequences $(1: 1: 1: 1: 1: 1)$ of treatment pathways. Three hundred and ninety-two participants will be recruited from secondary care DPNP centres in the UK. A list of participating centres can be found at the end of this paper. Recruitment is expected to take place over 12 months beginning in October 2017. Follow-up will continue for another 12 months.

The study contains an internal pilot with stop-go criteria to assess its feasibility. The recruitment and retention will be reviewed in relation to targets agreed with the funder after 6 months of recruitment. If met, an assessment of attrition to the trial is scheduled after 12 months of recruitment.

The study protocol was written in accordance with the Standard Protocol Items: Recommendations for Interventional Trials (SPIRIT) guidelines (see Additional file 1: SPIRIT Checklist).

\section{Participants}

A number of approaches will be used to identify potential participants: hospital database searches will be completed at each of the study centres; potential patients may be identified during routine hospital appointments at a study centre; the general practitioner (GP) patient registers at around 80 GP surgeries aligned to the study centres will be checked for patients with a diagnosis of diabetes and prescriptions for neuropathic pain medications; Participant Identification Centres (PIC) will be utilised; community podiatry services will be engaged to encourage referrals of potential patients, if applicable; details of the study will be advertised through the use of posters and leaflets in various clinics (for example, diabetes outpatient clinics or GP surgeries); the study will be advertised in a number of locations, such as on charity websites, in local libraries, local newspapers and via local radio stations to inform potential participants about the study.

Potentially eligible participants will be provided with the participant information sheet. Informed consent will be obtained by a medically qualified site investigator trained in Good Clinical Practice.

\section{Eligibility criteria \\ Inclusion criteria}

1. Participant aged $\geq 18$ years 
2. Neuropathic pain affecting both feet and / or hands for at least 3 months or taking pain medication for neuropathic pain for at least 3 months

3. Bilateral distal symmetrical neuropathic pain confirmed by the Douleur Neuropathique 4 (DN4) questionnaire at the screening visit [18]

4. Bilateral distal symmetrical polyneuropathy confirmed by a modified Toronto Clinical Neuropathy Score $(\mathrm{mTCNS})>5$ at the screening visit [19]

5. Stable glycaemic control $(\mathrm{HbA} 1 \mathrm{c}<108 \mathrm{mmol} / \mathrm{mol})$

6. Participants will have a mean total pain intensity of at least 4 on an 11-point numeric rating scale (NRS; with 0 being 'no pain' and 10 'worst pain imaginable') during 1 week off pain medications (baseline period)

7. Willing and able to comply with all the study requirements and be available for the duration of the study. This will be a 1-year study in which all participants will undergo all treatment pathways regardless of treatment response and this point will be made clear

8. Willing to discontinue current neuropathic-painrelieving medications

9. Informed consent form for study participation signed by participant

\section{Exclusion criteria}

1. Non-diabetic symmetrical polyneuropathies

2. History of alcohol/substance abuse which would, in the opinion of the investigator, impair their ability to take part in the study

3. History of severe psychiatric illnesses which would, in the opinion of the investigator, impair their ability to take part in the study

4. History of epilepsy

5. Contraindications to study medications

6. Pregnancy/breast feeding or planning pregnancy during the course of the study

7. Use of prohibited concomitant treatment that could not be discontinued

8. Use of high-dose morphine equivalent (>100 mg/day)

9. Liver disease (AST/ALT $>2$ times upper limit of normal)

10. Significant renal impairment (estimated glomerular filtration rate $(\mathrm{eGFR})<30 \mathrm{ml} / \mathrm{min} / 1.73 \mathrm{~m}^{2}$ )

11. Heart failure New York Heart Association (NYHA) $\geq$ class II

12. Clinically significant cardiac arrhythmias on 12-lead ECG or current history of arrhythmia

13. Patients with a recent myocardial infarction $(<6$ months prior to randomisation)
14. Postural hypotension (reduction of $>20 \mathrm{mmHg}$ )

15. Prostatic hypertrophy or urinary retention to an extent which would, in the opinion of the investigator, be a contraindication to the study medication

16. Patients with other painful medical conditions where the intensity of the pain is significantly more severe than their diabetic peripheral neuropathic pain (patients will not be excluded if the pain is transient in nature)

17. Any suicide risk as judged by the investigator or as defined by a score of $\geq 2$ on the suicide risk questionnaire

18. Significant language barriers which are likely to affect the participant's understanding of the medication schedule or ability to complete outcome questionnaires

19. Concurrent participation in another clinical trial of an investigational medicinal product

20. Major amputations of the lower limbs

21. Active diabetic foot ulcers

\section{Washout and baseline period}

After providing consent, participants will be instructed on how to washout neuropathic pain medication. The dose will be tapered for 3 days with complete washout for 1-4 days at the investigator's discretion. If the participant is on combination therapy then all drugs will be tapered at once. For participants taking 50-100-mg morphine equivalent the dose will be tapered over a period of up to 2 weeks.

Following the initial washout period, participants will enter the baseline period for 1 week. No neuropathic pain medication is permitted during this week with the exception of paracetamol. From the daily pain scores collected during the baseline period, a mean for the week will be determined and used in subsequent analysis.

\section{Interventions}

Treatment pathways

The OPTION-DM will study three treatment pathways:

- Amitriptyline supplemented with pregabalin (A-P Pathway)

- Duloxetine supplemented with pregabalin (D-P Pathway)

- Pregabalin supplemented with amitriptyline (P-A Pathway)

\section{Randomisation}

Participants will be centrally randomised in the study by the study team at site using the Clinical Trials Research Unit (CTRU) online randomisation system. All participants will receive all three treatment pathways (Table 1). 
Table 1 Treatment sequences $(A=$ amitriptyline, $P=$ pregabalin, $\mathrm{D}=$ duloxetine)

\begin{tabular}{llll}
\hline & $\begin{array}{l}\text { Treatment } \\
\text { pathway 1 }\end{array}$ & $\begin{array}{l}\text { Treatment } \\
\text { pathway 2 }\end{array}$ & $\begin{array}{l}\text { Treatment } \\
\text { pathway 3 }\end{array}$ \\
\hline Sequence 1 & A-P & D-P & P-A \\
Sequence 2 & A-P & P-A & D-P \\
Sequence 3 & D-P & A-P & P-A \\
Sequence 4 & D-P & P-A & A-P \\
Sequence 5 & P-A & A-P & D-P \\
Sequence 6 & P-A & D-P & A-P \\
\hline
\end{tabular}

Randomisation will determine the order in which they receive the treatment pathways. Participants will be assigned to one of the six sequences based on a predetermined randomisation schedule stratified by study site using permuted blocks.

\section{Treatment phases}

Each treatment pathway has two treatment phases (Fig. 1). During the first treatment phase, participants will receive monotherapy with the first-line treatment in the pathway. This will last for a total of 6 weeks, including the dose-titration phase.

At the week- 6 follow-up visit a decision will be taken to either continue on monotherapy or to add secondline treatment as combination therapy based on the 7-day average 24-h pain NRS score during the week preceding the study visit. Participants will be divided into 'responders' (pain score $\leq 3$ ) and 'non-responders' (pain score $>3$ ) and this will be used to guide treatment during the second treatment phase.

The second treatment phase will last for a total of 10 weeks. Non-responders will commence combination therapy with the addition of second-line treatment for 10 weeks, including the dose-titration phase. Responders will continue on monotherapy for the remainder of treatment phase 2, but this decision may be reversed up to week 13 in the event that a participant becomes a 'non-responder' later in the second treatment phase. The dose titration will follow the same schedule.

At the week-16 follow-up visit, participants will be advised to taper-down study medication (3 days) and stop the medication completely ( 4 days) before commencing the next treatment pathway. The taper dose will be one dose level below the maximum tolerated dose. Participants on dose level 1 will not require a taper dose and will stop study medication completely for 7 days. The first and second treatment phases will be repeated until the participant completes all three pathways.

\section{Dose titration}

There will be three dose levels for each drug and participants will always start on the lowest dose level of each drug. The schedule of dose escalation will be identical in each treatment pathway, see Fig. $2 a$ and b. Patients with renal insufficiency will receive a modified dosing schedule: eGFR will be measured at screening and at week 16 of each pathway and patients whose eGFR was 30-59 ml/ min at their most recent test will receive a lower dose of pregabalin. Pharmacy will be informed of the latest eGFR result with each prescription in order to ensure that the correct dose of pregabalin is dispensed.

During the first 2 weeks of each treatment phase, the dose will be escalated towards the maximum tolerated dose or maximum permitted dose, whichever is first, based on treatment response (based on the 24-h pain NRS score) and side effect profile.

During the weekly telephone calls and scheduled study visits, the research nurse will evaluate response to treatment and adverse effects to guide dose titration accordingly. If patients are receiving adequate pain relief (24-h pain NRS score $\leq 3)$ at dose level 1 or 2 then the dose will not be increased further. Patients will also be asked to rate any reported side effects. These will be graded (mild, moderate or severe) and whether side effects are tolerable or intolerable. Any severe or intolerable side effects will require a medication review (i.e. consider dose reduction or discontinuation).

\section{Switching treatment during a pathway}

At the week- 6 visit if there was no change in pain scores from the pre-treatment pathway (baseline), participants will switch to the second-line treatment in the treatment pathway.

If there is significant intolerance to first-line treatment; for example, due to side effects which are severe or which the patient describes as intolerable, participants can switch to the second-line treatment in the treatment pathway as a monotherapy. In this situation the switch can be made immediately, at any time, without the need to washout the first-line treatment. The second-line treatment will be continued as a monotherapy for the remainder of the treatment pathway; i.e. up to the week-16 visit. If there is significant intolerance to the second-line treatment in the pathway, the participant will stop the study treatment but will remain in the study for follow-up.

\section{Blinding}

OPTION-DM is a double-blind study and blinding of medication will be maintained with over-encapsulated drugs and matching placebos. The treating physician will be aware of the dose level but not the treatment itself. Due to the complex dosing schedule, the pharmacist at each study centre will be unblinded and a member of staff at Sheffield CTRU responsible for site monitoring will also be unblinded. 


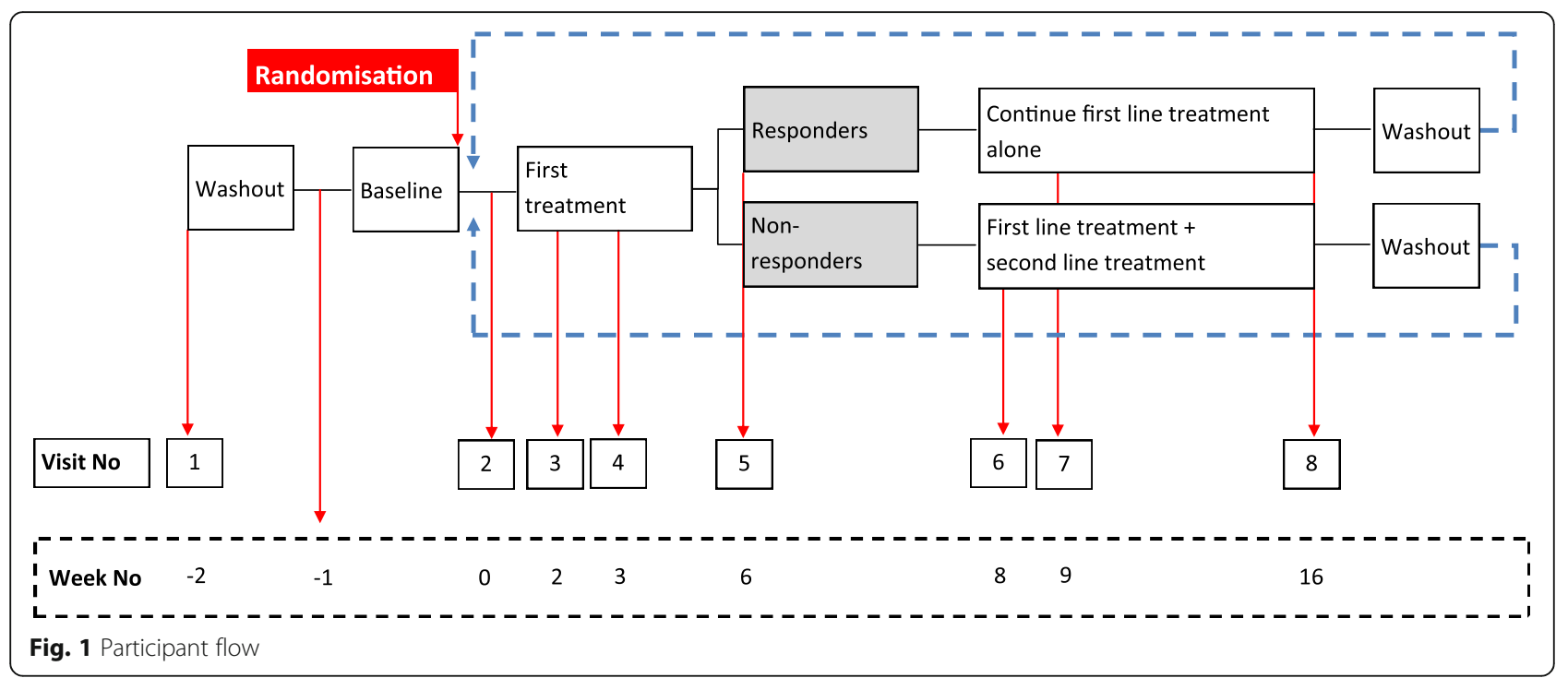

Emergency unblinding can be performed where the knowledge of the treatment allocation would change the participant's clinical management or to facilitate safety reporting to the regulatory authority and Research Ethics Committee.

\section{Adherence}

Participants will be provided with detailed guidance regarding how to take their study medication. This will be reinforced with written instructions and participants will be directed to complete a daily medication diary to record which doses they have taken.

Participants will be asked to return all bottles of study medication, including empty bottles and any unused medication. These will be reviewed and the remaining capsules counted to monitor adherence to study treatment. The study nurse will provide further guidance to participants if there is concern about adherence levels.

\section{Concomitant medications}

Participants will maintain their current schedule of treatment throughout the duration of the study. Changes to concomitant medications will be documented at each study visit. Participants may take paracetamol $1 \mathrm{~g}$ (up to a maximum dose of four times a day (QDS)) for pain throughout the study period.

The following concomitant medications are prohibited during the study period: opioid analgesia, capsaicin cream/high-dose capsaicin patches, lidocaine patches, anti-inflammatory medications (e.g. diclofenac, colecoxib), other antiepileptic medications (e.g. carbamazepine), other antidepressant medications (e.g. SSRIs, MAOIs), other neuropathic pain medications (e.g. venlafaxine, intravenously administered (IV) lignocaine, etc.), use of any medications that could lead to potentially serious interactions with study medications.

\section{Blood sample collection}

Blood samples will be stored for future research which may include genetic analysis. Samples will be obtained at the same time as other study blood samples from participants who have given additional (optional) consent. The blood will be frozen and stored locally before being shipped to a central laboratory.

\section{Study procedures}

The study assessment schedule (SPIRIT Figure; Fig. 3) below details the assessments required during the course of one treatment pathway. All participants will complete three treatment pathways and this schedule will be repeated from week 0 to week 16 until all three pathways are complete. Week 17 will only be relevant at the end of the final pathway.

\section{Primary outcome}

The primary outcome is the difference between 7-day average 24-h pain (evaluated at patient level) on an 11 -point NRS scale $(0=$ no pain and $10=$ worst pain imaginable) measured during the final follow-up week of the treatment cycle (week 16) among pathways. The NRS 24-h average pain is now considered the 'gold standard' for the assessment of neuropathic pain and has been employed in almost all well-designed neuropathic pain studies over the past 10 years $[15,20,21]$. 


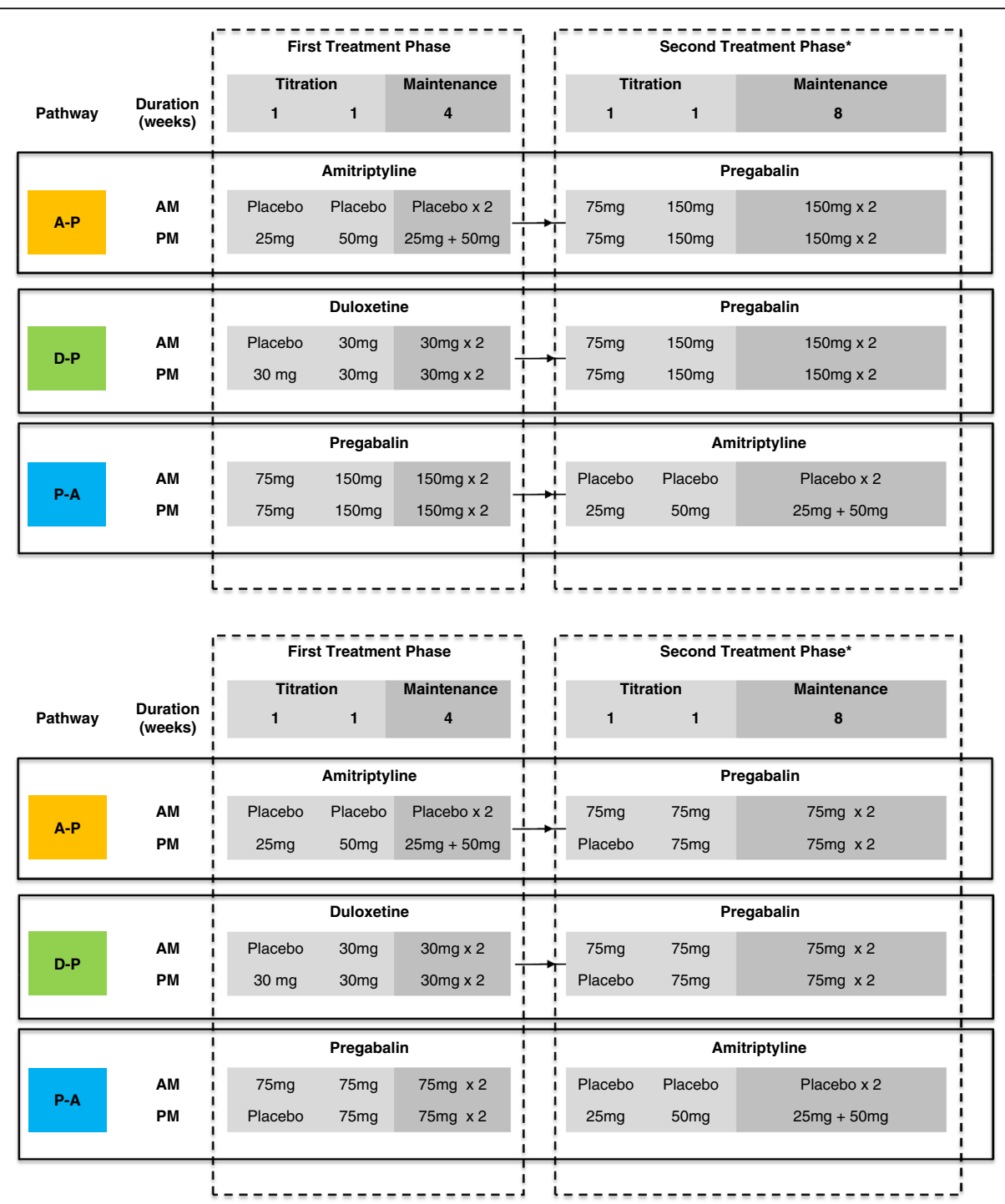

Fig. 2 a Dosing and titration schedule for each treatment pathway (standard pregabalin dosing, estimated glomerular filtration rate (eGFR) $\geq 60 \mathrm{ml}$ min). *Participants continue on the maintenance dose of drug from the first treatment phase for the duration of the second treatment phase. $\mathbf{b}$ Dosing and titration schedule for each treatment pathway (reduced pregabalin dosing, eGFR 30-59 m//min). *Participants continue on the maintenance dose of drug from the first treatment phase for the duration of the second treatment phase

\section{Secondary outcomes \\ Efficacy}

1. Difference between 7-day average 24-h pain (evaluated at patient level) on an 11-point NRS scale at week 6 among monotherapies

2. Difference between RAND short form 36 (RAND SF-36) physical mean scores (evaluated at patient level) at week 16 among pathways [22]

3. Difference between RAND SF-36 physical mean scores (evaluated at patient level) at week 6 among pathways [22]
4. Difference between RAND SF-36 mental mean scores (evaluated at patient level) at week 16 among pathways [22]

5. Difference between RAND SF-36 mental mean scores (evaluated at patient level) at week 6 among pathways [22]

6. Difference between Hospital Anxiety and Depression Scale (HADS) mean anxiety scores (evaluated at patient level) at week 6 among pathways [23].

7. Difference between Hospital Anxiety and Depression Scale (HADS) mean anxiety scores (evaluated at patient level) at week 16 among pathways [23] 


\begin{tabular}{|c|c|c|c|c|c|c|c|c|c|}
\hline \multirow{2}{*}{ Assessments } & \multirow{2}{*}{$\begin{array}{c}\text { Screening } \\
-2^{\mathrm{a}}\end{array}$} & \multicolumn{8}{|c|}{ Weeks from starting treatment pathway ${ }^{b}$} \\
\hline & & $0^{c}$ & $2^{c}$ & $3^{c}$ & $6^{c}$ & $8^{c, d}$ & $9^{c}$ & $16^{\mathrm{c}, \mathrm{e}}$ & 17 \\
\hline Informed consent & $\mathrm{x}$ & & & & & & & & \\
\hline Blood Tests ${ }^{\mathrm{gh}}$ & $\mathrm{x}$ & & & & & & & $\mathrm{x}$ & \\
\hline ECG & $x$ & & & & & & & & \\
\hline Medical History & $x$ & & & & & & & & \\
\hline Physical and neurological assessment & $\mathrm{x}$ & & & & & & & & \\
\hline modified Toronto Clinical Neuropathy Score (mTCNS) & $x$ & & & & & & & & \\
\hline Douleur Neuropathique 4 (DN4) & $x$ & & & & & & & & \\
\hline Suicidal risk questionnaire & $x$ & & & & & & & & \\
\hline Concomitant Medications & $\mathrm{x}$ & $\mathrm{x}$ & $\mathrm{x}$ & $\mathrm{x}$ & $\mathrm{x}$ & $\mathrm{x}$ & $\mathrm{x}$ & $\mathrm{x}$ & $\mathrm{x}$ \\
\hline Vital Signs' & $\mathrm{x}$ & & & & & & & $\mathrm{x}$ & \\
\hline Pregnancy Test (for women of child bearing potential) & & $x^{k}$ & & $\mathrm{x}$ & $\mathrm{x}$ & & $\mathrm{x}$ & $x$ & \\
\hline Randomisation (treatment allocation) & & $x^{k}$ & & & & & & & \\
\hline Dispense Study Medication & & $\mathrm{x}$ & $\mathrm{x}$ & $\mathrm{x}$ & $\mathrm{x}$ & $\mathrm{x}$ & $\mathrm{x}$ & $\mathrm{x}$ & \\
\hline Pain Diaries $^{\mathrm{j}}$ & $\mathrm{x}$ & $\mathrm{x}$ & $x$ & $x$ & $x$ & $x$ & $\mathrm{x}$ & $x$ & \\
\hline Tolerability scale & & $x^{k}$ & & & $x$ & & & $x$ & \\
\hline Brief Pain Inventory-Modified Short Form (BPI-MSF) & & $x^{k}$ & & & $x$ & & & $x$ & \\
\hline Insomnia Severity Index (ISI) & & $x^{k}$ & & & $\mathrm{x}$ & & & $\mathrm{x}$ & \\
\hline Neuropathy Pain Symptom Inventory (NPSI) & & $x^{k}$ & & & $\mathrm{x}$ & & & $\mathrm{x}$ & \\
\hline Hospital Anxiety and Depression Scale (HADS) & & $x^{k}$ & & & $\mathrm{x}$ & & & $\mathrm{x}$ & \\
\hline RAND Short Form 36 (RAND SF-36) & & $x^{k}$ & & & $\mathrm{x}$ & & & $\mathrm{x}$ & \\
\hline EQ-5D-5L & & $x^{k}$ & & & $\mathrm{x}$ & & & $\mathrm{x}$ & \\
\hline Client Service Receipt Inventory (CSRI) & & $x^{k}$ & & & $x$ & & & $x$ & \\
\hline Pain Catastrophising Scale (PCS) & & $x^{k}$ & & & & & & & \\
\hline Adverse Events Assessment & & $x^{\top}$ & $\mathrm{x}$ & $\mathrm{x}$ & $\mathrm{x}$ & $\mathrm{x}$ & $\mathrm{x}$ & $x$ & $\mathrm{x}$ \\
\hline Compliance Assessment & & $x^{\top}$ & $x$ & $x$ & $x$ & $\mathrm{x}$ & $x$ & $x$ & $\mathrm{x}$ \\
\hline Patient Global Impression of Change (PGIC) & & & & & & & & $x$ & \\
\hline
\end{tabular}

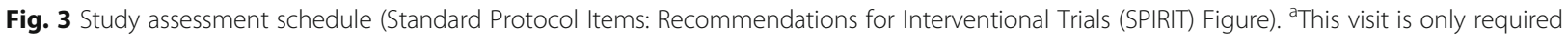
prior to randomisation, i.e. before starting the first treatment pathway. ${ }^{b}$ Between scheduled study visits, the research nurse will contact the participant by telephone each week (a minimum of once per week). The nurse will confirm compliance with medication and remind the participant to complete study diaries/questionnaires. ${ }^{C}$ Visits must normally be within \pm 2 days of the scheduled visit date. Scheduled visit dates relate to the date of the previous visit. Where this is impossible, e.g. due to bank holidays or patient availability. ${ }^{d}$ Week-8 visit only required for participants on combination treatment. ${ }^{e}$ At the week-16 visit, participants will be given instructions to tape-off the current study treatment. Visits from week 0 to week 16 will be repeated until all 3 pathways have been completed. ${ }^{f}$ Week 17 is only applicable following the final pathway. ${ }^{g}$ FBC, urea and electrolytes, liver function tests, glycosylated haemoglobin A1c and serum creatinine. ${ }^{\mathrm{h}}$ hole blood sample to be collected and stored for future research. The sample can be obtained at the same time as any scheduled blood test for the study. Please refer to the OPTIONDM Sample Collection Manual for details. ${ }^{i}$ Height (at week -2 only), weight, heart rate and blood pressure (lying and standing). ${ }^{j}$ To be completed by participants daily during the study, starting during the washout period. Pain scores may also be collected via daily text messages where participants have given additional consent for this. ${ }^{k}$ Only required at week 0 of pathway 1, i.e. randomisation visit. . Not required at week 0 of pathway 1, i.e. randomisation visit

8. Difference between Hospital Anxiety and Depression Scale (HADS) mean depression scores (evaluated at patient level) at week 6 among pathways [23]

9. Difference between Hospital Anxiety and Depression Scale (HADS) mean depression scores (evaluated at patient level) at week 16 among pathways [23]

10. Difference in proportion of patients having treatment success (30\%) at week 16 among pathways. Treatment success is defined as a reduction in 30\% value of 7 -day average NRS score at follow-up compared to baseline

11. Difference in proportion of patients having treatment success (50\%) at week 16 among pathways. Treatment success is defined as a reduction in $50 \%$ value of 7 -day average NRS score at follow-up compared to baseline

Difference in Brief Pain Inventory - modified short form (BPI-MSF) measure of pain interference with function total score (evaluated at patient level) at week 6 among pathways [24]
12. Difference in BPI-MSF measure of pain interference with function total score (evaluated at patient level) at week 16 among pathways [24]

13. Difference in Insomnia Severity Index (evaluated at patient level) total score at week 6 among pathways [25]

14. Difference in Insomnia Severity Index (evaluated at patient level) total score at week 16 among pathways [25]

15. Difference in Patient Global Impression of Change (evaluated at patient level) at week 16 among pathways [26]

16. Difference in proportion of care pathway preferred by participants at week 50

\section{Cost-effectiveness}

17. EuroQoL-5D-5 L: the EQ-5D is a routinely used generic HRQL instrument. It is the preferred instrument for assessing HRQL by NICE, and the newer five-level (EQ-5D-5 L) instrument offers increased sensitivity as opposed to the original three-level version [27] 
18. A modified version of the Client Service Receipt Inventory (CSRI): the CSRI is a routinely used instrument to capture health resource use and personal expenses. Unnecessary questions will be removed to reduce participant burden [28]

\section{Safety}

20. Frequency and proportion of patients reporting at least one Adverse Event for each of the pathway. Additionally, the relationship to intervention (Definite, Probable, Possible, Unlikely, Unrelated, Not assessable) will be reported (frequency and proportion)

21. Frequency and proportion of Adverse Events for each of the pathways

22. Listing of Adverse Events for each of the pathways

23. Frequency and proportion of patients reporting at least one Serious Adverse Event for each of the pathways. Additionally, these characteristics will be summarised (frequency and proportion): intensity (Mild, Moderate, Severe), relationship (Definite, Probable, Possible, Unlikely, Unrelated, Not assessable), is SUSAR, is Death

24. Frequencies of Serious Adverse Events for each of the pathways

25. Listing of Serious Adverse Events for each of the pathways

\section{Subgroup}

Neuropathic Pain Symptom Inventory (NPSI) questionnaire for subgroup analysis relating pain phenotype to treatment response [29]. There is emerging evidence that treatment response may be determined by a patient's pain phenotype [30-32]. In particular, these outcomes will be evaluated:

26. Difference between 'Burning (superficial) spontaneous pain' NPSI mean subscores (evaluated at patient level) at week 6 among pathways

27. Difference between 'Burning (superficial) spontaneous pain' NPSI mean subscores (evaluated at patient level) at week 16 among pathways

28. Difference between 'Pressing (deep) spontaneous pain' NPSI mean subscores - (evaluated at patient level) at week 6 among pathways

29. Difference between 'Pressing (deep) spontaneous pain' NPSI mean subscores - (evaluated at patient level) at week 16 among pathways

30. Difference between 'Paroxysmal pain' NPSI mean subscores - (evaluated at patient level) at week 6 among pathways
31. Difference between 'Paroxysmal pain' NPSI mean subscores - (evaluated at patient level) at week 16 among pathways

32. Difference between 'Evoked pain' NPSI mean subscores - (evaluated at patient level) at week 6 among pathways

33. Difference between 'Evoked pain' NPSI mean subscores - (evaluated at patient level) at week 16 among pathways

34. Difference between 'Paresthesia/dysaesthesia' NPSI mean subscores - (evaluated at patient level) at week 6 among pathways

35. Difference between 'Paresthesia/dysaesthesia' NPSI mean subscores - (evaluated at patient level) at week 16 among pathways

36. Difference between NPSI mean total scores (evaluated at patient level) at week 6 among pathways

37. Difference between NPSI mean total scores (evaluated at patient level) at week 16 among pathways

\section{Patient-perceived tolerability}

38. Difference between tolerability (evaluated at patient level) on an 11-point NRS scale at week 16 among pathways-.

39. Difference between tolerability (evaluated at patient level) on an 11-point NRS scale at week 6 among monotherapies

\section{Sample size}

A 1-point change in an individual on the NRS scale is considered a minimum clinically important difference [33]. Hence, the proportion of patients improving by at least 1 point would seem a suitable outcome. However, we have based the sample size calculation on a continuous outcome, the mean change between groups in order to maintain power [34]. We have chosen a mean change at the population level of 0.5 points between groups based on the effect size previously reported for comparison of two active interventions for neuropathic pain in a crossover study [35]. Based on Normal Distribution Theory we estimate that a 0.5 -point shift in population means will lead to an additional $8 \%$ of individual patients achieving a 1-point improvement [36]. We have also used a conservative, Bonferroni-corrected significance of $1.67 \%$ in order to retain an overall $5 \%$ false-positive probability for finding a significant pairwise comparison. Using a within-patient SD of 1.65 [35], an alpha of 0.0167 and $90 \%$ power, we require 294 evaluable patients [37]. Assuming a 25\% dropout rate 392 patients will be randomised to ensure that 294 patients are expected to complete the study. 


\section{Withdrawals}

An individual participant may stop treatment early for any of the following reasons:

- Unacceptable toxicity

- Withdrawal of consent for treatment by the participant

- Inter-current illness which prevents further treatment

- Any alteration in the participant's condition which justifies the discontinuation of treatment in the investigator's opinion

- Pregnancy

Participants will be followed up as per the trial schedule until the end of the current treatment pathway, provided they are willing. A discussion will also take place to clarify whether the participant is discontinuing all study treatment or whether they wish to return for the next treatment pathway.

When a participant stops treatment in the OPTIONDM study, they will return to their usual care provider for treatment outwith the study. At the end of the trial, when the final analysis has been completed, the CTRU will provide participating sites with the unblinded treatment allocations for each of their participants. The site staff will be responsible for contacting each of the participants to notify them of the treatment allocations. It will then be a clinical decision between the participant and their usual care provider as to which treatment they receive.

\section{Data collection and management}

Participant confidentiality will be respected at all times and the principles of the UK Data Protection Act (DPA) will be followed. The study will use the CTRU's in-house data management system (Prospect) for the capture and storage of study-specific participant data. Access to Prospect is controlled by usernames and encrypted passwords, and a comprehensive privilege management feature will be used to ensure that users have access to only the minimum amount of data required to complete their tasks. A member of staff at each site will enter data from source documents into the study-specific Prospect database when available. After data have been entered, electronic validation rules are applied to the database on a regular basis; discrepancies are tracked and resolved through the Prospect database. Questionnaires are self-completed by participants and entered onto the study database by the research nurse. Data will be stored and managed in accordance with CTRU Standard Operating Procedures (SOPs).

\section{Statistical analysis}

The statistical analysis will be reported according to Consolidated Standards of Reporting Trials (CONSORT) guidelines [38] and using an intention-to-treat approach as the primary analysis. As three pairwise comparisons will be performed, all statistical tests will be two-tailed at a $1.67 \%$ significance level. The primary outcome and other continuous outcomes will be analysed using a random-effects model with participant, treatment, sequence and period entered into the model. Participant will be entered as a random term. Contrasts will be used to evaluate the difference in means. Three $98.33 \%$ confidence intervals for the difference on treatment effect will be reported as well as the associated $P$ value.

In case of missing data, the missing data mechanism will be explored and multiple imputation may be applied as a sensitivity analysis as appropriate. Other sensitivity analyses will be performed in order to evaluate the robustness of the primary analyses [39].

A logistic regression will be undertaken to analyse binary outcomes using a model similar to that for the continuous outcomes. Differences between treatment groups will be reported as odds ratios with associated 98.33\% confidence intervals and $P$ values. Full details of the statistical analyses will be specified in a detailed Statistical Analysis Plan.

\section{Monitoring}

Conduct of this study will be governed by three committees. An independent Trial Steering Committee (TSC) will oversee the conduct of the trial. An independent Data Monitoring and Ethics Committee (DMEC) will monitor participant safety. A Trial Management Group (TMG) will be responsible for the day-to-day running of the trial. The roles and responsibilities of the groups are included in the group charter or terms of reference.

CTRU will undertake monitoring visits at each investigator site before, during and after the trial. Central monitoring will also be utilised to review data, consent forms and accountability logs.

Details of Adverse Events will be collected at each study visit or telephone call. Serious Adverse Events will be assessed by the local investigator and reported to Sheffield CTRU within $24 \mathrm{~h}$.

\section{Ethics and dissemination}

Ethical approval for the study was given by Yorkshire and the Humber - Sheffield Research Ethics Committee (reference number: 16/YH/0459). Any protocol amendments will be reviewed and approved by the Ethics Committee and regulatory authority as applicable before being notified to all relevant parties.

The results of the trial will be disseminated in peer-reviewed scientific journals and clinical and academic conferences. A lay summary of the results will be sent directly to participants. The results will also be freely available via the funding body's journal website 
[40] and a summary will be published on the Sheffield CTRU website.

\section{Patient public involvement}

The Diabetes PPI Panel at Sheffield Teaching Hospitals reviewed the study at the proposal development stage. They were supportive of the proposal including the study design and they contributed to the choice of endpoints for the study. In particular, they were pleased with the efficient crossover design as participants will receive active treatment during all treatment phases. Although the duration of the study is long, they felt that participants are more likely to remain in the study as active treatment is received. The panel were later involved in the development of the patient information sheet, consent form and study medication diary. We also have a patient representative as a member of the TSC; therefore, we will have ongoing patient involvement in the management of the study.

\section{Discussion}

DPNP is a distressing and disabling condition which is often intractable to treatment. Unfortunately current treatment only achieves meaningful pain relief in two out of three patients. Despite much research there are no current or emerging treatments that alter the natural course of the disease. This study is timely as it addresses an important clinical need by providing evidence as to which is the most clinically beneficial and cost-effective treatment pathway for DPNP.

\section{Why examine treatment pathways?}

The examination of a treatment pathway as a whole is the most efficient and applicable to current UK clinical practice. This is because most patients are started on monotherapy and will require a second agent added in combination within a few months [41]. Only a very small minority will either have sufficient benefit from monotherapy and will not need another agent, or will not tolerate monotherapy (or monotherapy is completely ineffective) and will be switched to another agent. Thus, OPTIONDM, which will examine the whole treatment pathway, will capture more clinically relevant outcomes than artificially designed, head-to-head monotherapy or combination studies. Hence, the outcomes of this study will be readily generalisable to current UK clinical practice.

\section{Why exclude gabapentin?}

There is clear rationale for not studying two $\alpha-2-\delta$ agonists (pregabalin and gabapentin) as:

1. The evidence for gabapentin is only derived from one reasonable-quality RCT (4-week titration and 4-week treatment phase) [42] compared to eight
RCTs in pregabalin and evidence supported by meta-analysis [15]

2. Gabapentin is a thrice daily drug

3. Gabapentin, unlike pregabalin does not have linear pharmacokinetics and requires a long titration period of up to 2 months [43] to avoid toxicity

\section{Which treatment pathways?}

We will not examine the pathway of pregabalin supplemented by duloxetine because of the COMBO-DN findings [44]. In this study, there was no difference in pain reduction if pregabalin was added to duloxetine or vice versa [44]. However, duloxetine was superior to pregabalin as an initial treatment, is a once daily preparation and is also the cheaper option in the UK. There is thus a good rationale for starting patients on duloxetine and then adding pregabalin in combination. Finally, as both amitriptyline and duloxetine are antidepressants there is little rationale for combining both.

\section{Efficient design with 16-week treatment pathways}

This will be an efficiently designed head-to-head, crossover RCT [21] with each patient undergoing all pathways. The duration of monotherapy in each pathway is at least 6 weeks, an adequate duration to assess treatment effect and whether combination therapy is indicated [21, 43]. The subsequent 10-week combination therapy in patients with partial benefit from monotherapy will be adequate to assess stabilised treatment outcomes [44]. The COMBO-DN study used fixed-dose-titration regimens regardless of treatment response. This resulted in a dropout rate of $17 \%$ during monotherapy and $12 \%$ during combination therapy [44]. The present trial is a pragmatic RCT employing a flexible dosing regimen to achieve maximum-tolerated doses based on individual responses; we envision that this will reduce the dropout rate. The use of rescue medication, frequent clinic and telephone contacts and the need for active therapy we envision will further reduce dropout rates. Completion rates will be monitored on an ongoing basis.

\section{Trial status}

The current protocol is version 7.0, 1 February 2018. The study began recruiting in November 2017 and is estimated to be completed in October 2018. Follow-up will continue for a further 12 months. We anticipate that the results will be available in early 2020 .

\section{Additional file}

Additional file 1: Standard Protocol Items: Recommendations for Interventional Trials (SPIRIT) Checklist. (DOC 119 kb) 


\section{Abbreviations}

BPI-MSF: Brief Pain Inventory - modified short form; CSRI: Client Service Receipt Inventory; CTRU: Clinical Trials Research Unit; DN4: Douleur Neuropathique 4; DPNP: Diabetic peripheral neuropathic pain; eGFR: Estimated glomerular filtration rate; HADS: Hospital Anxiety and Depression Scale; ISI: Insomnia Severity Index; mTCNS: Modified Toronto Clinical Neuropathy Score; NICE: National Institute for Health and Care Excellence; NPSI: Neuropathy Pain Symptom Index; NRS: Numeric rating scale; PCS: Pain Catastrophising Scale; PGIC: Patient Global Impression of Change; PIC: Participant Identification Centre; QoL: Quality of life; RAND SF36: RAND short form 36; RCT: Randomised controlled trial; SOP: Standard operating procedure

\section{Acknowledgements}

The OPTION-DM Group: Satyan Rajbhandari, David Bennett, Rajiv Gandhi, Edward Jude, Gerry Rayman, Prashanth Vas, Ravikanth Gouni, Didier Bouhassira, Michelle Horspool, Martin Johnson and Tracey Young. The clinical sites taking part in the study are: Sheffield Teaching Hospitals NHS Foundation Trust, Tameside Hospital NHS Foundation Trust, Ipswich Hospital NHS Trust, Nottingham University Hospitals NHS Trust, Oxford University Hospitals NHS Foundation Trust, Countess of Chester Hospital NHS Foundation Trust, Royal Bournemouth and Christchurch Hospitals NHS Foundation Trust, Harrogate and District NHS Foundation Trust, Lancashire Teaching Hospitals NHS Trust and Aintree University Hospital NHS Foundation Trust. The Trial Steering Committee members are: Andrew Rice (chair), Ralf Baron, Sarah Brown, Arthur Durrant, Nanna Finnerup, Frances Game, Roger Knaggs and Blair Smith. The Data Monitoring and Ethics Committee members are: Troels Jensen (Chair), Zoe Hoare and Martin Rutter.

\section{Funding}

The research is funded by the National Institute for Health Research (NIHR) Health Technology Assessment (HTA) programme (project number: 15/35/ 03). The funder has reviewed the research protocol but will have no role in data collection, analysis, data interpretation, report writing or in the decision to submit the report for publication.

\section{Sponsor}

Sheffield Teaching Hospitals.

\section{Authors' contributions}

DS and JP contributed equally to this paper. DS, JP, DW, SJ, OB, CC, MB, AL, $H B, L S, K S$ and ST contributed to the design of the protocol and drafted or critically reviewed the manuscript. All authors read and approved the final manuscript.

\section{Ethics approval and consent to participate}

The protocol was approved by Yorkshire and the Humber - Sheffield Research Ethics Committee (16/YH/0459) and received Medicines and Healthcare products Regulatory Agency (MHRA) clinical trials authorisation (21,304/0262/001). Written informed consent will be obtained from all participants prior to any study-specific procedures.

\section{Consent for publication}

Not applicable.

\section{Competing interests}

The authors declare that they have no competing interests.

\section{Publisher's Note}

Springer Nature remains neutral with regard to jurisdictional claims in published maps and institutional affiliations.

\section{Author details}

'Department of Oncology and Human Metabolism, Medical School, University of Sheffield, Sheffield, UK. ${ }^{2}$ Clinical Trials Research Unit, University of Sheffield, Sheffield, UK. ${ }^{3}$ Medical Statistics Group, School of Health and Related Research, University of Sheffield, Sheffield, UK. ${ }^{4}$ Sheffield Teaching Hospitals NHS Foundation Trust, Sheffield, UK.
Received: 20 March 2018 Accepted: 3 October 2018

Published online: 22 October 2018

\section{References}

1. Abbott C, Malik R, Van Ross ERE, Kulkarni J, Boulton AJM. Prevalence and characteristics of painful diabetic neuropathy in a large community-based diabetic population in the U.K. Diabetes Care. 2011:34:2220-4.

2. Davies M, Brophy S, Williams R, Taylor A. The prevalence, severity, and impact of painful diabetic peripheral neuropathy in type 2 diabetes. Diabetes Care. 2006;29:1518-22.

3. Veves A, Backonja M, Malik R. Painful diabetic neuropathy: epidemiology, natural history, early diagnosis, and treatment options. Pain Med. 2008:9:660-74

4. Boulton A, Vinik A, Arezzo J. Diabetic neuropathies: a statement by the American Diabetes Association. Diabetes Care. 2005;28:956-62.

5. Galer BS, Gianas A, Jensen MP. Painful diabetic polyneuropathy: Epidemiology, pain description, and quality of life. Diabetes Res Clin Pract. 2000;47:123-8.

6. Zelman DC, Brandenburg N, Gore M. Sleep impairment in patients with painful diabetic peripheral neuropathy. Clin J Pain. 2006;22:681-5. https:// doi.org/10.1097/01.ajp.0000210910.49923.09.

7. Gore M, Brandenburg N, Dukes E, Hoffman DL, Tai KS, Stacey B. Pain severity in diabetic peripheral neuropathy is associated with patient functioning, symptom levels of anxiety and depression, and sleep. J Pain Symptom Manag. 2005;30:374-85.

8. Gore M, Brandenburg N, Hoffman D, Tai K-S, Stacey B. Burden of illness in painful diabetic peripheral neuropathy: the patients' perspectives. J Pain. 2006:7:892-900. https://doi.org/10.1016/j.jpain.2006.04.013.

9. Tolle T, Xu X, Sadosky AB. Painful diabetic neuropathy: a cross-sectional survey of health state impairment and treatment patterns. J Diabetes Complicat. 2006;20:26-33.

10. National Institute for Health and Care Excellence. Neuropathic pain in adults: pharmacological management in non-specialist settings. NICE Clin Guidel. 2013; https://www.nice.org.uk/guidance/cg173.

11. Moore R, Derry S, Aldington D, Cole P, Wiffen PJ. Amitriptyline for neuropathic pain in adults. Cochrane Database Syst Rev. 2015;7:CD008242. https://doi.org/10.1002/14651858.CD008242.pub3.www.cochranelibrary.com.

12. Lunn $M$, Hughes $R$, Wiffen $P$. Duloxetine for treating painful neuropathy, chronic pain or fibromyalgia (Review). Cochrane Database Syst Rev. 2014;1. https://www.cochranelibrary.com/cdsr/doi/10.1002/14651858.CD007115. pub3/full

13. Moore R, Straube S, Wiffen PJ, Derry S, McQuay HJ. Pregabalin for acute and chronic pain in adults. Cochrane Database Syst Rev. 2009;3:CD007076.

14. Moore R, Wiffen P, Derry S, Mcquay H. Gabapentin for chronic neuropathic pain and fibromyalgia in adults (Review). Cochrane Database Syst Rev. 2011; 3. https://www.cochranelibrary.com/cdsr/doi/10.1002/14651858.CD007938. pub2/full

15. Freeman R, Durso-DeCruz E, Emir B. Efficacy, safety, and tolerability of pregabalin treatment for painful diabetic peripheral neuropathy: findings from seven randomized, controlled trials across a range of doses. Diabetes Care. 2008;31:1448-54

16. Sultan A, Gaskell H, Derry S, Moore RA. Duloxetine for painful diabetic neuropathy and fibromyalgia pain: systematic review of randomised trials. BMC Neurol. 2008;8:29. https://doi.org/10.1186/1471-2377-8-29.

17. Wong $M$, Chung JWY, Wong TKS. Effects of treatments for symptoms of painful diabetic neuropathy: systematic review. BMJ. 2007;335:87. https://doi. org/10.1136/bmj.39213.565972.AE.

18. Bouhassira D, Attal N, Alchaar H, Boureau F, Brochet B, Bruxelle J, et al. Comparison of pain syndromes associated with nervous or somatic lesions and development of a new neuropathic pain diagnostic questionnaire (DN4). Pain. 2005;114:29-36.

19. Bril V, Tomioka S, Buchanan RA, Perkins BA. Reliability and validity of the modified Toronto Clinical Neuropathy Score in diabetic sensorimotor polyneuropathy. Diabet Med. 2009;26:240-6

20. Quilici S, Chancellor J, Löthgren M, Simon D, Said G, Le TK, et al. Metaanalysis of duloxetine vs. pregabalin and gabapentin in the treatment of diabetic peripheral neuropathic pain. BMC Neurol. 2009;9:6. https://doi.org/ 10.1186/1471-2377-9-6.

21. Dworkin RH, Turk DC, Peirce-Sandner S, Burke LB, Farrar JT, Gilron I, et al. Considerations for improving assay sensitivity in chronic pain clinical trials: 
IMMPACT recommendations. Pain. 2012;153:1148-58. https://doi.org/10. 1016/j.pain.2012.03.003.

22. Ware JE Jr, Gandek B. Overview of the SF-36 Health Survey and the International Quality of Life Assessment (IQOLA) Project. J Clin Epidemiol. 1998:51:903-12.

23. Zigmond A S, Snaith RP. The Hospital Anxiety and Depression Scale. Acta Psychiatr Scand. 1983;67:361-70.

24. Cleeland CS, Ryan KM. Pain assessment: global use of the brief pain inventory. Ann Acad Med Singap. 1994;23:129-38.

25. Bastien $\mathrm{CH}$, Vallières $\mathrm{A}$, Morin $\mathrm{CM}$. Validation of the Insomnia Severity Index as an outcome measure for insomnia research. Sleep Med. 2001;2:297-307. https://doi.org/10.1016/S1389-9457(00)00065-4.

26. Guy W. ECDEU assessment manual for psychopharmacology. Rockville: Natl Inst Ment Heal Psychopharmacol Res Branch; 1976. p. 217-22. 313-31, 534-7

27. Devlin N, Shah K, Feng Y, Mulhern B, van Hout B. Valuing health-related quality of life: an EQ-5D-5L value set for England. 2016. http://eprints. whiterose.ac.uk/97964/1/An_EQ-5D-5L_Value_Set_for_England_DP_Final.pdf

28. Beecham J, Knapp M. Costing psychiatric interventions. In: Measuring mental health needs. Edited by Thornicroft G. London: Gaskell; 2001. p. 20024.

29. Bouhassira D, Attal N, Fermanian J, Alchaar H, Gautron M, Masquelier E, et al. Development and validation of the Neuropathic Pain Symptom Inventory. Pain. 2004:108:248-57.

30. Demant D, Lund K, Vollert J, Maier C, Segerdahl M, Finnerup N, et al. The effect of oxcarbazepine in peripheral neuropathic pain depends on pain phenotype: a randomised, double blind, placebo-controlled phenotypestratified study. Pain. 2014;155:2263-73.

31. Bouhassira D, Wilhelm S, Schacht A, Perrot S, Kosek E, Cruccu G, et al. Neuropathic pain phenotyping as a predictor of treatment response in painful diabetic neuropathy: data from the randomized, double-blind, COMBO-DN study. Pain. 2014;155:2171-9. https://doi.org/10.1016/j.pain.2014.08.020.

32. Marchettini $P$, Wilhelm $S$, Petto $H$, Tesfaye $S$, Tölle T, Bouhassira D, et al. Are there different predictors of analgesic response between antidepressants and anticonvulsants in painful diabetic neuropathy? Eur J Pain. 2016;20:472-82.

33. Dworkin RH, Turk DC, Wyrwich KW, Beaton D, Cleeland CS, Farrar JT, et al. Interpreting the Clinical Importance of Treatment Outcomes in Chronic Pain Clinical Trials: IMMPACT Recommendations. J Pain. 2008;9:105-21.

34. Senn S, Julious $S$. Measurement in clinical trials: a neglected issue for statisticians? Stat Med. 2009;28:3189-209.

35. Gilron I, Bailey JM, Tu D, Holden RR, Weaver DF, Houlden RL. Morphine, gabapentin, or their combination for neuropathic pain. N Engl J Med. 2005; 352:1324-34. https://doi.org/10.1056/NEJMoa042580.

36. Julious S, Walters SJ. Estimating effect sizes for health-related quality of life outcomes. Stat Methods Med Res. 2014;23:430-9. https://doi.org/10.1177/ 0962280213476379.

37. Julious SA. Tutorial in biostatistics: sample sizes for clinical trials with Normal data. Stat Med. 2004;23:1921-86.

38. Moher D, Hopewell S, Schulz KF, Montori V, Gotzsche PC, Devereaux PJ, et al. CONSORT 2010 explanation and elaboration: updated guidelines for reporting parallel group randomised trials. Int I Surg. 2012;10:28-55.

39. Thabane L, Mbuagbaw L, Zhang S, Samaan Z, Marcucci M, Ye C, et al. A tutorial on sensitivity analyses in clinical trials: the what, why, when and how. BMC Med Res Methodol. 2013;13:92. https://doi.org/10.1186/14712288-13-92.

40. NIHR Journals Library. https://www.journalslibrary.nihr.ac.uk/HTA/\#/.

41. Margolis JM, Princic N, Smith DM, Abraham L, Cappelleri JC, Shah SN, et al. Development of a novel algorithm to determine adherence to chronic pain treatment guidelines using administrative claims. J Pain Res. 2017;10:327-39.

42. Backonja M, Beydoun A, Edwards K, Schwartz S, Fonseca V, Hes M, et al. Gabapentin for the symptomatic treatment of painful neuropathy in patients with diabetes mellitus. A randomized controlled trial. JAMA. 1998;280(21):1831-6. https://doi.org/10.1001/jama.280.21.1831

43. Dworkin R, O'Connor A, Audette J, Baron R, Gourlay G, Haanpaa M, et al. Recommendations for the pharmacological management of neuropathic pain: an overview and literature update. Mayo Clin Proc. 2010;85(3 Suppl): S3-14 https://doi.org/10.4065/mcp.2009.0649.

44. Tesfaye S, Wilhelm S, Lledo A, Schacht A, Tölle T, Bouhassira D, et al. Duloxetine and pregabalin: high-dose monotherapy or their combination? the 'COMBO-DN study' - A multinational, randomized, double-blind, parallel-group study in patients with diabetic peripheral neuropathic pain. Pain. 2013;154:2616-25.

\section{Ready to submit your research? Choose BMC and benefit from}

- fast, convenient online submission

- thorough peer review by experienced researchers in your field

- rapid publication on acceptance

- support for research data, including large and complex data types

- gold Open Access which fosters wider collaboration and increased citations

- maximum visibility for your research: over $100 \mathrm{M}$ website views per year

At BMC, research is always in progress.

Learn more biomedcentral.com/submissions 